\title{
Wheat Leaf Rust Fungus: RIMAPS Analysis to Detect Germ-tube Orientation Pattern.
}

\author{
L.M. Setten ${ }^{1}$, N. Lendoiro ${ }^{2}$ and E.A. Favret ${ }^{3,4}$ \\ ${ }^{1}$ Lab. Microscopía y Capacidades Afines, CICVyA, INTA, Hurlingham, Argentina. \\ ${ }^{2}$ Fac. Cs. Exactas, Químicas y Naturales, Universidad de Morón, Morón, Argentina. \\ ${ }^{3}$ Instituto de Suelos, CIRN, INTA. Hurlingham. Argentina. \\ ${ }^{4}$ CONICET. Buenos Aires. Argentina.
}

Wheat is severely affected by a destructive disease, leaf rust, caused by fungus, Puccinia triticina E. All over the world, wheat leaf rust reduces crop yield up to $10 \%$. When the uredospore reaches the leaf surface develops germ-tubes, which are directed towards the stomata. The germ-tube grows and elongates perpendicular to the long axis of the epidermal cells, this orientation is considered to be response to stimuli from the host (Fig. 1), such us physical and chemical features [1]. The topography of the leaf surface has been proposed by several investigators as a major factor that can influence the direction of the germ-tube growth. Our research work is based on determining which of these topographic parameters influence the development of the germ-tubes, by using artificial surfaces with known topographies. For that purpose it is necessary to quantify the angular orientation of the germ-tube pattern as a first step. The present paper shows how RIMAPS can be used as a tool to detect this orientation pattern. The RIMAPS technique consists basically of rotating the image using algorithms of commercial software and calculating the $\mathrm{x}$-step of the two-dimensional Fourier transform for each $y$-line of the new image obtained after rotation. Averaged power spectra are obtained for each angular position. The corresponding maximum values are plotted as a function of rotation angle. The maxima of the RIMAPS spectrum indicate main angular directions of the topographic pattern [2]. For this study, two samples of an inert material, polystyrene (PS), were used. The surface of one sample was micro-structured with direct laser interference patterning technique mimicking the direction of the epidermal cells (Fig. 2), the other was smooth [3]. Both samples were inoculated with rust spore suspension. The surfaces were examined with a VP-SEM FEI Quanta 250. Afterwards, for the RIMAPS studies hand-made drawings from the SEM images were done in order to copy only the pattern of the germ-tubes observed on the PS surfaces (Fig. 3-4), eliminating any artifacts of the surface sample. The RIMAPS spectra are seen in figures 5 and 6 . Figure 5 indicates two main maxima $\left(4^{\circ}, 142^{\circ}\right)$. The direction of the micro-structure corresponds to $90^{\circ}$. Therefore, RIMAPS shows that the main orientation $\left(4^{\circ}\right)$ of the germ-tube pattern is almost perpendicular to the micro-structure. The other maximum $\left(142^{\circ}\right)$ needs to be properly explained with more studies. The RIMAPS spectrum of figure 6 determines several main maxima $\left(18^{\circ}, 75^{\circ}, 112^{\circ}, 147^{\circ}\right)$. In this case the orientation of the germ-tube pattern has no preferential direction. Presently, other parameters of the spectrum are also been considered, such as the integral of the curve which increases with the number of germ-tubes directions, e. $g$., figure 5 has an integral of 118,5 and figure 6 an integral of 173,2. The difference observed between the RIMAPS spectra would support that the surface topography is one factor that influences the orientation of germ-tube and would be a method to quantify the orientation [4].

References

[1] G Hu and FHJ Rijkenberg, Mycol. Res. 102 (1998), p. 391-399.

[2] NO Fuentes and EA Favret, Journal of Microscopy 206 (2002), p. 72-83.

[3] A Lasagni et al., Proc. of SPIE 8968 (2014), p. 1-9.

[4] The authors acknowledge Andrés Lasagni, Francisco Sacco and Lorena La Fuente for their collaboration. 


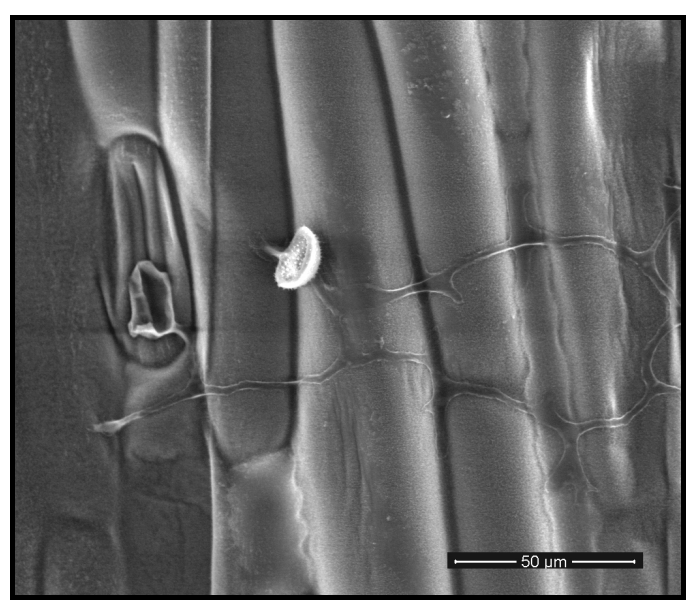

Figure 1. SEM of a rust spore showing the orientation of germ-tube on the leaf wheat.

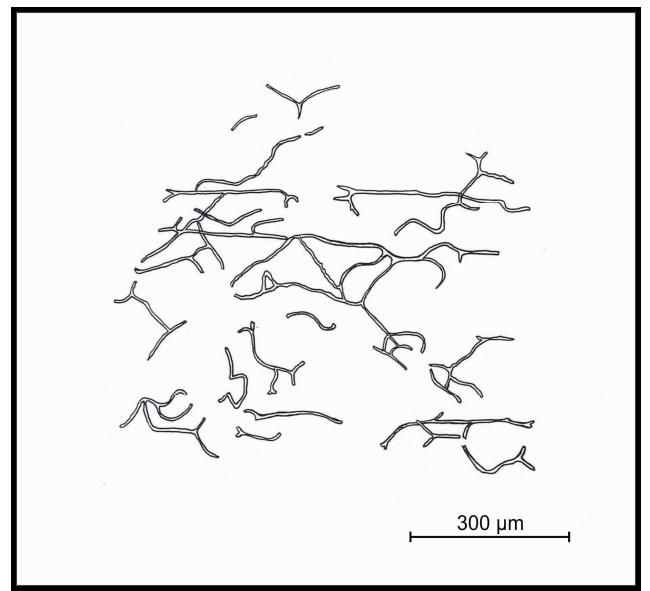

Figure 3. Hand-made drawing copy of the germ-tubes on the microstructured PS.

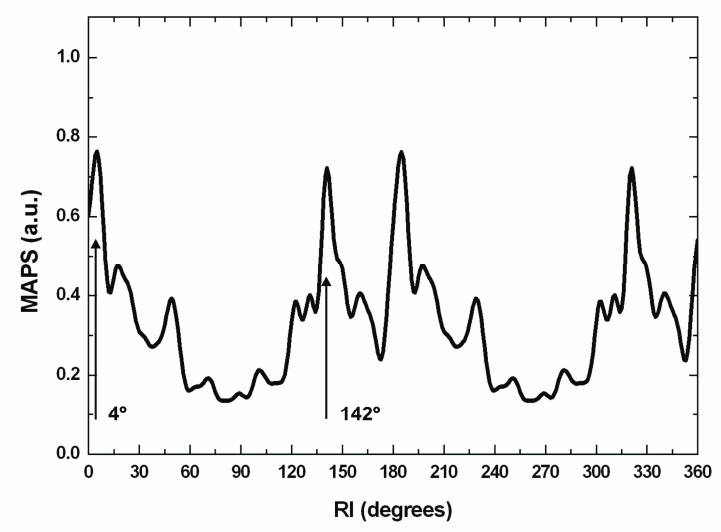

Figure 5. RIMAPS spectrum of Figure 3.

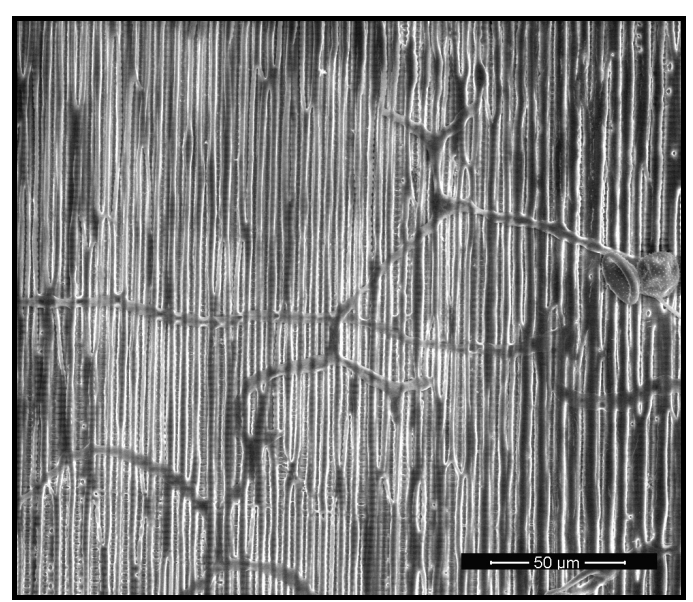

Figure 2. SEM of a rust spore showing the orientation of germ-tube on the microstructured PS.

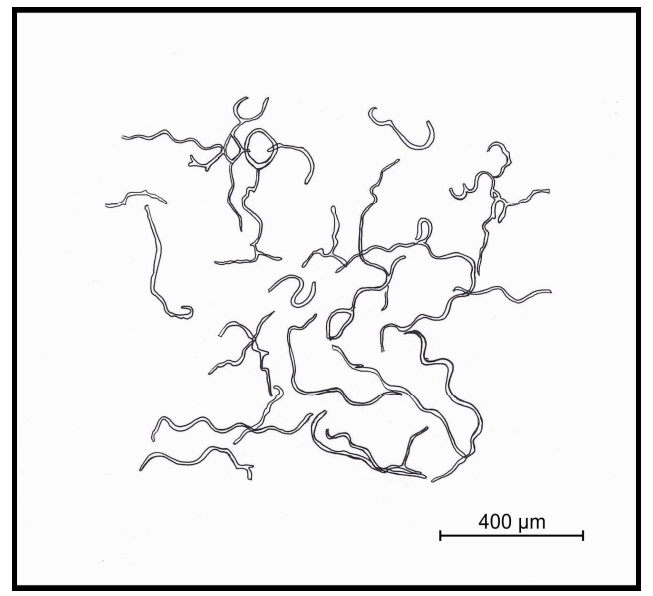

Figure 4. Hand-made drawing copy of the germ-tubes on the smooth PS.

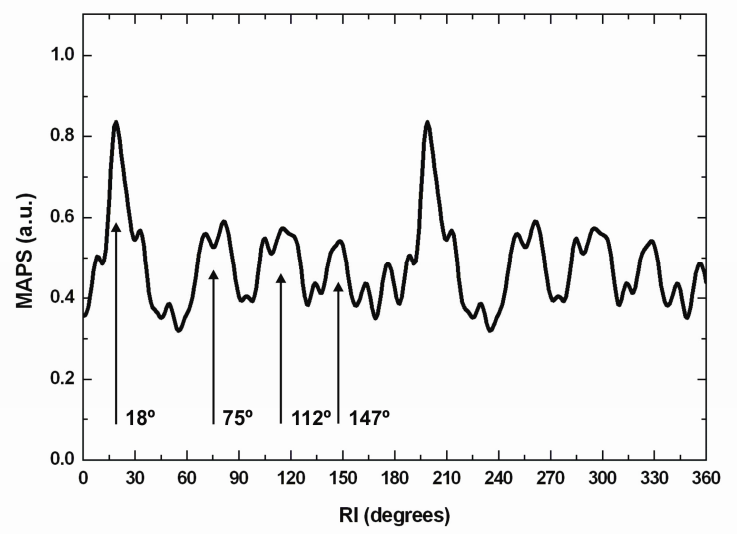

Figure 6. RIMAPS spectrum of Figure 4. 